\title{
Comparing the Incidence of Postdural Puncture Headache After Diagnostic Lumbar Puncture due to 22 Gauge Standard Versus 25 Gauge Atraumatic Needle : A Randomized Controlled Trial
}

\author{
Ashok Kumar Phani ${ }^{*}$ \\ Somen Chowdhury ${ }^{2}$ \\ Priyatosh Das ${ }^{3}$ \\ Farid Uddin Ahmed ${ }^{4}$ \\ Md. Humayun Kabir ${ }^{5}$ \\ Md.Ruhul Amin ${ }^{6}$ \\ Pradip Kumar Kayasthagir ${ }^{7}$ \\ Md. Hassanuzzaman
}

'250 Bed Chattogram General Hospital Chattogram, Bangladesh.

${ }^{2}$ Department of Neurology

Chattogram Maa Shisu-O-General Hospital Chattogram, Bangladesh.

${ }^{3}$ Department of Microbiology

Chittagong Medical College

Chattogram, Bangladesh.

${ }^{4}$ Department of Community Medicine

Chittagong Medical College

Chattogram, Bangladesh.

${ }^{5}$ Chittagong Medical College Hospital Chattogram, Bangladesh.

${ }^{6}$ Department of Neurology Rangpur Medical College Hospital

Rangpur, Bangladesh.

${ }^{7}$ Department of Neurology

Chittagong Medical College Hospital

Chattogram, Bangladesh.

\section{*Correspondence to:}

Ashok Kumar Phani

Medical Officer

250 Bed General Hospital

Chattogram, Bangladesh.

Mobile : +8801715847647

Email : ashokphani5@gmail.com

Date of Submission : 21.12 .2020

Date of Acceptance $\quad$ : 25.01 .2021

www.banglajol.info/index.php/CMOSHMCJ

\begin{abstract}
Background: There is growing evidence that the incidence of Postdural Puncture Headache (PDPH) after Lumber Puncture (LP) with the smaller, non-cutting needle is less. Nevertheless, larger, cutting needles are still widely used for this procedure in Bangladesh. The aim of this study was to compare the incidence and severity of $P D P H$ between $22 \mathrm{G}$ standard needle and $25 \mathrm{G}$ atraumatic needle for diagnostic $L P$ in patients with neurological symptoms.
\end{abstract}

Methods and materials: This single-blind, randomized, controlled trial was carried out in Department of Neurology, Chittagong Medical College Hospital, during February 2017 to January 2018. One hundred consecutive patients admitted in Neurology ward fulfilled the set inclusion and exclusion criteria were enrolled in the study and randomly divided into two equal groups: Group A (LP was done with $22 \mathrm{G}$ standard needle) and Group B (LP was done with $25 \mathrm{G}$ atraumatic needle). The incidence and severity of PDPH was interviewed on day 5 following $L P$.

Results: Effective sample size was 99 (50 in Group A and 49 in Group B) as one patient was dropped out. Both groups were similar in terms of baseline sociodemographic and clinical characteristics. LP was successful in first attempt in 25 (50\%) and 28 (57.8\%) patients in Group A and Group B respectively. Incidence of $P D P H$ was significantly higher in Group A than Group B. Severity of PDPH was also significantly higher in Group $A$ than in Group $B$ with regards to presence of moderate degree of PDPH. Analgesics use was significantly less in Group $B$ in comparison to Group A. Absolute adjusted risk of mild to moderate PDPH with $25 \mathrm{G}$ atraumatic needles was reduced by 3.74 times (95\% Cl: 1.22-11.44) compared to 22 $G$ standard needle.

Conclusion: $25 G$ atraumatic needles significantly reduced the incidence and severity of PDPH as compared to $22 \mathrm{G}$ standard needle. So, using $25 \mathrm{G}$ atraumatic needle would be beneficial for diagnostic $L P$ procedure.

Key words: PDPH; Diagnostic LP; 25G atraumatic needle; 22G standard needle.

\section{INTRODUCTION}

Lumbar puncture (LP) is a technique to sample Cerebrospinal Fluid (CSF) as a window into brain pathology. The procedure involves introducing a needle into the subarachnoid space of the lumbar sac, at a level safely below the spinal cord ${ }^{1}$. Despite modern neuroimaging techniques, LP remains an important diagnostic tool ${ }^{2}$. Postdural Puncture Headache (PDPH) is one of the most common complications of LP occurring within 5 days of the procedure ${ }^{2}$. PDPH is characteristically located over frontal and occipital region radiating to the neck and shoulder, aggravated by upright posture and relieved by lying down. It might be associated with nausea, vomiting, tinnitus, vertigo, dizziness, paresthesia of scalp, limb pain, visual disturbances, cranial nerve palsies etc. Symptoms usually start within 48 hours in $66 \%$ cases and 
within 3 days in more than $90 \%$ cases $^{3,4}$. Although PDPH is usually self limiting and non fatal, its postural nature prevents the patients from performing routine activities. It prolongs hospital stay and cost as well. These patients require additional psychological support and therapeutic measures. These justified the search for preventive measures that decrease incidence of $\mathrm{PDPH}^{3}$.

Various preventive measures has been tried to reduce the incidence of PDPH. Reducing the size of the spinal needle has made direct impact on PDPH. The incidence is $2-12 \%$ with a 26G Quincke needle, $\sim 40 \%$ with a $22 \mathrm{G}$ needle, up to $25 \%$ with a $25 \mathrm{G}$ needle and $<2 \%$ with a $29 \mathrm{G}$ needle or smaller ones ${ }^{5-9}$. But failure of the affect with $29 \mathrm{G}$ or smaller needles is quite common due to technical difficulties ${ }^{8}$. Although proven to reduce the occurrence of $\mathrm{PDPH}$, the usage of small, non-cutting needles is still not widespread in neurology departments ${ }^{10}$. In a UK survey, a cutting needle was used in over $70 \%$ of neurology units, and only two of 48 units reported using a gauge smaller than $22 \mathrm{G}^{11}$. In a US survey, the use of non-cutting needles was even lower, as only $2 \%$ of the responding neurologists reported that they routinely used this type of needle ${ }^{12}$. In Bangladesh the scenario is not different. $25 \mathrm{G}$ atraumatic needle is not used commonly for diagnostic LP in neurology ward. The reasons given for the continued use of larger cutting needles vary from economical and practical concerns to a lack of up-to-date knowledge ${ }^{10-12}$.

There is scarcity of data regarding comparison of 25 gauge atraumatic needle as opposed to a 22 gauge standard needle for diagnostic LP in our country. In this context, this study was conducted to compare the incidence and severity of PDPH following diagnostic LP done with 25 gauge atraumatic needle and 22 gauge standard needle.

\section{MATERIALS AND METHODS}

This randomized controlled trial was conducted in the Neurology Department of Chittagong Medical College Hospital, Chattogram, Bangladesh, from February 2017 to January 2018. One hundred consecutive admitted patients, age's $\geq 18$ years and needed elective diagnostic LP was included in the study. Unconscious patient $(\mathrm{GCS}<8)$ with a history of chronic headache requiring analgesics, known or suspected cases of idiopathic intracranial hypertension, patients with features of raised intracranial pressure, patients having hemorrhagic disorder, localized skin infection, pathological localized deformity in lumbar region were excluded. After consenting, the patients were randomized to lumbar puncture with either a $22 \mathrm{G}$ standard needle (Quincke) or $25 \mathrm{G}$ atraumatic needle (Pencil-point). Randomization was done by a computer generated code stored in opaque envelopes that were serially numbered and sealed.

A single trained neurologist performed all LP procedures with the patient in the left lateral position and patients were blinded of the type of needle used for the procedure. However, because the different needles have different structures, the physician knew which needle was used and could not be blinded to the needle. All patients rested in bed for at least four hours after the procedure, and fluid intake was encouraged.
The patients' age, sex, body mass index, and presumptive diagnoses were recorded. During the procedure the operators documented the number of attempts, the time involved with each, and opening and closing pressure, the volume of cerebrospinal fluid removed, and the volume of local anaesthetic used.

Five day after LP, the patients were reassessed directly (For admitted patients) or over telephone (Discharged patients). The incidence of PDHP was recorded and their severity graded as mild, moderate, or severe. Data regarding associated symptoms puncture site pain, treatment history and hospital readmission were also recorded.

Continuous variables were reported either as mean \pm Standard Deviation (SD) or median (Range) and categorical variables were reported as frequency (Percentage). Baseline characteristics were compared by either Student's t-test or Mann-Whitney $\mathrm{U}$ test whichever was applicable for continuous data or the Chisquare test or Fisher's exact test for categorical data. For PDPH the relative risk, absolute risk reduction, relative risk reduction, and numbers needed to treat for benefit, with $95 \%$ confidence intervals were calculated. Logistic regression analysis was performed to determine the independent association between needle types PDHP. Statistical significance was defined as $\mathrm{p}<0.05$ and confidence interval was set at $95 \%$ level. The data were analyzed for significance with SPSS version 23.

The study protocol was approved by the Ethical Review Committee of Chittagong Medical College, and written informed consent was obtained from all patients.

\section{RESULTS}

One hundred patients were initially enrolled and randomized in the study. Out of 100 patients 99 completed the study. All LP indications were neurological (Peripheral neuropathy, demylinating disease, Tubercular meningitis, Acute Transverse Myelitits and Neurosyphilis). Age, sex, BMI, pulse, blood pressure, GCS, number of attempts and CSF volume collected were not significantly different between the two groups (Table I). Technical variables related to LP procedure were parallel cutting axis of the needles, reinsertion of stylet, number of attempts needed for successful LP, change of needle, use of local anesthetics and failure of LP. Reinsertion of stylet was done in every cases and parallel cutting axis of needle was used for $22 \mathrm{G}$ standard Group but not applicable for $25 \mathrm{G}$ atraumatic Group. There were no failed LPs (Not shown in Table).

Table I : Baseline characteristics of patients.

\begin{tabular}{lrrr} 
Parameters & \multicolumn{2}{c}{ Needle type for LP } & p value \\
& 22 G standard \\
$(\mathrm{n}=50)$ & $\begin{array}{r}25 \mathrm{G} \text { atraumatic } \\
(\mathrm{n}=49)\end{array}$ \\
Age (Years) & $37.90( \pm 14.90)$ & $37.49( \pm 18.26)$ & $0.903^{\dagger}$ \\
Male & $31(62.0)$ & $27(55.1)$ & $0.489^{*}$ \\
Weight $(\mathrm{Kg})$ & $56.26( \pm 10.19)$ & $54.43( \pm 8.01)$ & $0.437^{\dagger}$ \\
Height (meter) & $1.61( \pm 0.07)$ & $1.59( \pm 0.07)$ & $0.130^{\dagger}$ \\
BMI in $\left(\mathrm{Kg} / \mathrm{m}^{2}\right)$ & $21.69( \pm 3.41)$ & $21.49( \pm 2.93)$ & $0.086^{\dagger}$ \\
Pulse (/Minute) & $82.08( \pm 9.09)$ & $79.63( \pm 6.67)$ & $0.130^{\dagger}$ \\
SBP (mmHg) & $123.30( \pm 13.84)$ & $118.27( \pm 11.44)$ & $0.052^{\dagger}$ \\
DBP (mmHg) & $77.20( \pm 7.23)$ & $75.61( \pm 7.54)$ & $0.287^{\dagger}$ \\
Glasgow coma scale & $14.58( \pm 1.11)$ & $14.86( \pm 0.87)$ & $0.169^{\dagger}$ \\
Volume of CSF drawn $(\mathrm{ml})$ & $2(1.5-2.5)$ & $2(1.5-2.5)$ & $0.430^{\dagger}$ \\
No. of LP attempts & $1.5(1-3)$ & $1(1-3)$ & $0.341^{\ddagger}$ \\
\hline
\end{tabular}


Data are presented either as mean $( \pm \mathrm{SD})$ frequency (Percentage) or median (Range) as appropriate. BMI: Body Mass Index, SBP: Systolic Blood Pressure, DBP: Diastolic Blood Pressure. ${ }^{*}$ Not significant by Chi-square test: ${ }^{\dagger}$ : Not significant by independent sample $\mathrm{t}$ test. ${ }^{\star}$ Not significant by Mann Whitney U test.

Incidence of PDPH was $15(30 \%)$ and $6(12.2 \%)$ respectively in $22 \mathrm{G}$ standard Group and $25 \mathrm{G}$ atraumatic Group and it was significant statistically $(\mathrm{p}=0.031$ ). This gave an absolute risk reduction of $17.76 \%$ and $59.2 \%$ relative risk reduction for PDPH when $22 \mathrm{G}$ standard needle was used comrade to $25 \mathrm{G}$ atraumatic needle. Puncture site pain was similar in both groups Though mild degree of PDPH was similar in both groups, moderate PDPH was significantly higher in $22 \mathrm{G}$ standard Group rhan $25 \mathrm{G}$ atraumatic Group (24\% versus $2.0 \%$; $\mathrm{p}=0.001$ ).

Table II : Characteristics of PDPH and puncture site pain.

\begin{tabular}{|c|c|c|c|}
\hline \multirow[t]{2}{*}{ Parameters } & \multicolumn{2}{|c|}{ Needle type for LP } & p value \\
\hline & \multicolumn{3}{|c|}{$22 \mathrm{G}$ standard $(\mathrm{n}=50) 25 \mathrm{G}$ atraumatic $(\mathrm{n}=49)$} \\
\hline Incidence of PDPH & $15(30)$ & $6(12.2)$ & $0.031^{\dagger}$ \\
\hline \multicolumn{4}{|l|}{ Severity of PDPH } \\
\hline Mild PDPH & $3(6.0)$ & $5(10.3)$ & $0.443^{*}$ \\
\hline Moderate PDPH & $12(24.0)$ & $1(2.1)$ & $0.001^{\dagger \dagger}$ \\
\hline Analgesia for PDPH & $12(24.0)$ & $1(2.1)$ & $0.001^{\dagger \dagger}$ \\
\hline \multicolumn{4}{|l|}{ Associated symptoms } \\
\hline Neck stiffness & $5(10.0)$ & $1(2.1)$ & $0.097^{*}$ \\
\hline Hearing disturbance & $1(2.0)$ & $0(0)$ & $0.325^{*}$ \\
\hline Puncture site pain & $10(20.0)$ & $7(14.2)$ & $0.487^{*}$ \\
\hline
\end{tabular}

Data are presented as frequency (Percentage), ${ }^{\dagger}:$ Significant by Chi-square test, ${ }^{\dagger}$ :Significant by Fischer Exact test, $*$ :Not significant by Fischer Exact test.

Binary logistic regression (Unadjusted and adjusted) analysis were conducted to determine the factors associated with PDPH. It revealed that, only needle type came out as the only independent factor associated with PDPH. Patients who were tapped with $22 \mathrm{G}$ were 3.47 times higher likely to develop PDPH than those who were tapped with $25 \mathrm{G}$.

Table III : Crude and adjusted association between PDPH and other risk factors of PDPH.

\begin{tabular}{|c|c|c|c|c|c|c|}
\hline \multirow{2}{*}{\multicolumn{2}{|c|}{ Factors }} & \multirow{4}{*}{$\begin{array}{r}\text { PDPH } \\
\text { n, }(\%) \\
18(24.0) \\
3(12.7)\end{array}$} & \multicolumn{3}{|c|}{ Relative risk for PDPH, RR (95\% CI for RR) } & \multirow[b]{2}{*}{ p value } \\
\hline & & & Crude RR & p value & Adjusted RR & \\
\hline \multirow[t]{2}{*}{ Age } & $<50$ years & & Reference & 0.104 & Reference & 0.248 \\
\hline & $\geq 50$ years & & $2.25(0.84-5.99)$ & & $1.5(0.78-4.85)$ & \\
\hline \multirow[t]{2}{*}{ Sex } & Male & $9(11.5)$ & Reference & & Reference & 0.465 \\
\hline & Female & $12(29.5)$ & $2.21(0.59-8.28)$ & 0.239 & $2.01(0.57-6.48)$ & \\
\hline \multirow[t]{2}{*}{ BMI } & $<20 \mathrm{Kg} / \mathrm{m}^{2}$ & $17(23.6)$ & Reference & & Reference & 0.483 \\
\hline & $\geq 20 \mathrm{Kg} / \mathrm{m}^{2}$ & $4(15.4)$ & $1.67(0.51-5.52)$ & 0.397 & $1.01(0.21-5.35)$ & \\
\hline \multirow[t]{2}{*}{ Attempts } & 1 attempt & $12(22.6)$ & Reference & & Reference & 0.719 \\
\hline & $>1$ attempt & $9(19.6)$ & $1.20(0.45-3.18)$ & 0.709 & $1.10(0.56-4.32)$ & \\
\hline Needle & $25 \mathrm{G}$ & $6(12.2)$ & Reference & 0.031 & Reference & 0.021 \\
\hline type & $22 \mathrm{G}$ & $15(30.0)$ & $3.07(1.08-8.75)$ & & $3.47(1.22-11.44)$ & \\
\hline
\end{tabular}

RR: Relative Risk, CI: Confidence Interval.

\section{DISCUSSION}

Despite the presence of accumulated evidence regarding the beneficial role of small-bore needles compared to larger diameter cutting needles in diagnostic LP, in our setting larger-bore needles ( $22 \mathrm{G}$ standard needle) are still being used for this purpose. It was probably due to shortage of local evidence regarding the utility of small bore needle. This trial was conducted to compare the incidence and severity of PDPH following diagnostic LP done either by $22 \mathrm{G}$ standard or $25 \mathrm{G}$ atraumatic needle. The current study confirmed a reduced incidence of mild to moderate PDPH with $25 \mathrm{G}$ atraumatic needles compared to $22 \mathrm{G}$ standard needles in a typical group of neurological patients. Our result is similar to Strupp et al Engedal et al and Lavi et al who were able to confirm the findings that using a smaller, non-cutting needle significantly reduces the risk of PDPH after adjusting for potential confounders (Age, sex, BMI) ${ }^{13-15}$. Histological studies have shown that the atraumatic needles cause a smaller dural defect, which theoretically should lead to a lower incidence of $\mathrm{PLPH}^{10}$. Our study clearly supports this view.

Regarding the severity of PDPH, all of the events were either mild or moderate degree in severity in the current study. In 22 $\mathrm{G}$ standard needle group frequency of mild and moderate degree of PDPH was $6 \%$ and $24 \%$ respectively compared to $10.28 \%$ and $2 \%$ in $25 \mathrm{G}$ atraumatic needle group. Thomas et al find the proportion of mild, moderate and severe PDPH in atraumatic needle were $14.28 \%, 14.28 \%$ and $14.28 \%$ respectively and in standard needle group the corresponding values were $10.20 \%, 16.66 \%$ and $37.5 \%$ respectively ${ }^{16}$. In our study no patients in either group had severe type of PDPH. This could be due to small sample size and experience of operator as single operator allowed in this study in contrast to seven operators allowed in above study ${ }^{16}$.

Thomas et al reported a slightly higher rate of multiple attempts or failure to perform the procedure when using the atraumatic needle. However, this was not a significant finding, but was consistent with the subjective assessment that the operators found the non-cutting needle more difficult to use ${ }^{16}$. Of particular interest, the use of an introducer cannula was left to the operator's discretion in this study, whereas in our study it was obligatory ${ }^{16}$. In the present study, there is higher rate of multiple attempts to perform the procedure when using standard needle. Engedal et al showed same type of reports ${ }^{17}$. Multiple attempts were performed among those having BMI $>25 \mathrm{~kg} / \mathrm{m}^{2}$. Birnbach et al showed same type of result. Interestingly, the need for multiple attempts did not increase the risk of PDPH in the present study ${ }^{12}$. Engedal et al also showed multiple attempts did not increase the risk of $\mathrm{PDPH}^{14}$. This is consistent with the pathogenesis of the disease, such that only a successful attempt, penetrating the dura, is capable of inducing PDPH. In addition, accounting for the fact that in vitro investigation has confirmed that both smaller needle size and a non-cutting design reduce CSF leakage, these observations seriously question the classic notion, that the risk of PDPH is somehow dependent on the skill or the experience of the operator ${ }^{14}$. 
Incidence of PDPH showed a statistical non significant increasing trend among younger patients and female patients in the current study. These findings are almost similar with that of Engedal et al who had noticed that young age and female gender are risk factors for $\mathrm{PDPH}^{14}$. The present study demonstrated a trend toward increased prevalence of PDPH with lower BMI. However, the study did not find a significant association between PDPH and other clinical factors, potentially due to their minor influence and the relatively small number of patients in the study.

Regarding puncture site pain in this study, there was no significant difference between the two groups. Lavi et al showed same type of result ${ }^{15}$. Patients undergoing diagnostic LP with standard needle required more analgesia than those undergoing the procedure with atraumatic needle in the current study which was in line with the findings of Engedal et $\mathrm{al}^{14}$.

After adjusting for all confounders the patients who were tapped with $22 \mathrm{G}$ standard needles were 3.74 times more likely to have PDPH than those who were tapped with $25 \mathrm{G}$ atraumatic needls (95\% CI: 1.22 -11.4).
The strength of the current study was that the impact of technical variables on the PDPH risk was minimized almost to zero, as the same physician performed all LPs using the same technique. Furthermore, there were no intergroup differences in patient characteristics, thus preventing a possible adverse effect of these factors on the results. However, there were some limitations which should be considered while utilizing the study findings. Although patients were blinded to the needle type used in LP procedures but, it was not possible for investigator. Since outcome measures were self reported by a predesigned case record form, a rather simple score for assessing the degree of headache was chosen in this study. Moreover, comparison of the cost effectiveness of two types of needle was not addressed in the study.

\section{CONCLUSION}

The current study find that $25 \mathrm{G}$ atraumatic needle significantly reduced the incidence of PDPH in comparison to $22 \mathrm{G}$ standard needles during diagnostic LP in patients with neurological disorder without any failure rate.

\section{DISCLOSURE}

All the authors declared no competing interest. 


\section{REFERENCES}

1. Niemantsverdriet E, Struyfs H, Duits FH, Teunissen C, Engelborghss. Techniques, contraindications and complications of CSF collection procedures. In: Deisenhammer F, Sellebjerg F, Teunissen CE, Tumani H (eds). Cerebrospinal Fluid in Clinical Neurology. New York: Springer. 2015;35-57.

2. Headache Classification Committee of the International Headache Society (IHS). The International Classification of Headache Disorders, 3rd edition (beta version). Cephalalgia. 2013;33(9):629-808.

3. GpDeo. Review Article. Post Dural Puncture Headache. Journal of Chitwa Medical College. 2013;3(3): 5-10.

4. Christophe LW, christo P, Richman JM, Hsu W. Postdural Puncture Headache: An overview. The International Journal of Pain Medicine and Palliative Care. 2004;3(2):53-58.

5. Kuntz KM, Kokmen E, Stevens JC, Miller P, Offord KP, Ho MM. Post-lumbar puncture headaches: experience in 501 consecutive procedures. Neurology. 1992;42(10):1884-1887.

6. Kwak KH. Headache after dural puncture. Korean J Anesthesiol. 2017; 70(2): 136-143.

7. Flaatten H, Rodt SA, Vamnes J, Rosland J, Wisborg T, Koller ME. Postdural puncture headache. A comparison between 26- and 29-guage needles in young patients. Anaesthesia. 1989; 44(2): 147-149.

8. Babu DD, Chandar DD, Prokash CS, Balasubramanian S, Kumar KS. Evaluation of Post Dural Puncture Headache Using Various sizes of Spinal Needles. International Journal of Scientific Study. 2015;3(9):9-11.

9. Geurts JW, Haanschoten MC, Van Wijk RM, Kraak H, Besse TC. Post-dural puncture headache in young patients. A comparative study between the use of 0.52mm (25-guage) and 0.33mm (29-guage) spinal needles. ActaAnaesthesiolscand. 1990;34:350-353.

10. Arendt K, Demaerschalk BM, Wingerchuk DM, Camann W. Atraumatic lumbar puncture needles: after all these years, are we still missing the point? Neurologist. 2009;15(1):17-20.

11. Serpell MG, Haldane GJ, Jamieson DR, Carson D. Prevention of headache afterlumbar puncture: questionnaire survey of neurologists and neurosurgeons inUnited Kingdom. BMJ. 1998;316(7146):1709-1710.

12. Birnbach DJ, Kuroda MM, SternmanD,Thys DM. Use of atraumaticspinalneedles among neurologists in the United States. Headache 2001;41(4):385-390.

13. Strupp M, Schueler O, Straube A, Von Stuckrad-Barre S, Brandt T. "Atraumatic" Sprotte needle reduces the incidence of post-lumbar puncture headaches. Neurology. 2001;57(12):2310-2312.

14. Engedal TS, Ording H, Vilholm OJ. Changing the needle for lumbar punctures: results from a prospective study. Clin Neurol Neurosurg. 2015;130:74-79.

15. Lavi R, Yarnitsky D, Rowe JM, Weissman A, Segal D, Avivi I. Standard vs atraumatic Whitacre needle for diagnostic lumbar puncture: a randomized trial. Neurology. 2006;67(8):1492-1494.

16. Thomas SR, Jamieson DR, Muir KW. Randomised controlled trial of atraumatic versus standard needles for diagnostic lumbar puncture. BMJ. 2000;321(7267):986-990.

17. Engelborghs S, Niemantsverdriet E, Struyfs H, Blennow K, Brouns R, Comabella M et al. Consensus guidelines for lumbar puncture in patients with neurological diseases. Alzheimers Dement (Amst). 2017;8:111-126. 